\title{
Mechanical Characterization of the Heat Affected Zone of Gold Wirebonds Using Nanoindentation
}

\author{
M. Shah ${ }^{1}$, K. Zeng ${ }^{2}$, A.A.O. Tay ${ }^{3}$ and S. Suresh ${ }^{4}$ \\ Singapore-MIT Alliance, National University of Singapore \\ 2 Institute of Materials Research and Engineering, Singapore \\ Department of Mechanical Engg., National University of Singapore \\ Department of Materials Science and Engineering, Massachusetts Institute of Technology (MIT), Cambridge,USA
}

\begin{abstract}
With increasing miniaturization in microelectronics the wirebonds used in IC packages are witnessing a thrust towards fine pitch wirebonding. To have a precise control over loop height of the wirebond for fine pitch wirebonding, it is imperative to do mechanical characterization of the wirebond. The present work studies the mechanical properties of gold wire and wirebond using nanoindentation. The wirebond specimen surface was planarized using mechanical polishing.
\end{abstract}

The loop height of the gold wirebond is directly proportional to the length of the heat affected zone (HAZ) above the ball of gold wirebond. Metallographic preparation of gold wirebond cross section reveals the presence of undesirable coarse grain structure in HAZ due to recrystallization and grain growth in the gold wire adjacent to the ball. The recrystallization temperature of our gold wire was found using D.S.C. to be $340.66^{\circ} \mathrm{C}$. The doping elements present in the gold wire used, were identified using TOF-SIMS. Nanoindentation of the gold wire was done at different maximum loads to observe the hardness variation with load. The nanoindentation of gold wirebond has confirmed a $\mathrm{v}$-shaped hardness profile in the HAZ. The hardness minima for the particular gold wire used with a ball size ratio of 2.4 was observed at distance of $160-170 \mu \mathrm{m}$ from the neck of the ball. The elastic modulus was found to vary randomly and to be independent of the microstructure in the wirebond. A yield stress profile based on empirical hardness-yield strength correlation has been predicted for the gold wirebond.

Keywords-nanoindentation, wirebonding, heataffected zone (HAZ), fine-pitch, recrystallization, DSC, hardness, SIMS, ball bond, gold wire

\section{INTRODUCTION}

The growing complexity of silicon integrated circuit chips and their minaturization has resulted in higher I/O (InputOutput) pad density per chip leading to requirement for closer wirebond to wirebond spacing (fine pitch). The pitch is defined as the distance between the centers of two adjacent bonded wires and the trend is to reduce this gap as much as possible. There are three wire parameters which can be varied to get finer-pitch assembly: (1)smaller ball sizes (2) thinner wire diameter and (3) lower loop height. While the reduced bondability plagues the smaller ball sizes, the problem with using thinner wires is the occurrence of wire sweep which is due to lack of sufficient stiffness and rigidity in thin wires. For a fixed clearance between the capilliary and the wirebond, if the wire loop height is reduced the pitch between the wirebonds can be reduced.

The present work studies the factors influencing the loop height primarily by the use of nanoindentation. However during wirebonding, the wire does not necessarily have the same loop profile as capillary's trajectory [1]. The wire bends in the loop according to its own stiffness and rigidity and the loop height depends upon the mechanical characteristics of the Heat Affected Zone (HAZ) adjacent to the ball bonded to pad on the chip. Generally, the longer the heat affected zone, the higher is the loop height. Besides it has been known for quite some time, mostly from the wirebond pull-tests that the weakest part in the entire wirebond lies in the portion of wirebond just adjacent to the ball. Thus it is necessary to perform mechanical characterization of the HAZ region of gold wirebond and study its impact on loop profile.

The extremely small dimension of the gold wire

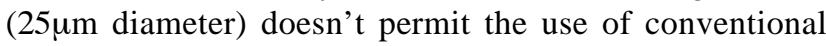
hardness testers for mechanical characterization. The present work uses a novel technique of low load depth sensing indentation i.e. Nanoindentation to characterize the wirebond. Besides this, the wire recrystallization characteristics are studied by use of Differential Scanning Calorimetry(DSC) and the doping elements in the bonding wire are identified using TOF-SIMS. Nanoindentation, being a depth sensing indentation technique, can also measure elastic properties besides the hardness. The nanoindentation tests have been conducted in the load controlled mode and tests have been conducted under different maximum loads on the plain bonding wire. The variation of the mechanical properties of the gold wirebond in the heat affected zone has been studied and the factors responsible for these are discussed. These findings are backed by microstructure analysis and metallurgical details of the ball-bond.

\section{SPECIMEN DESCRIPTION}

For nanoindentation testing, the specimen surface 
metallography. The shallower the indentation depth, the more smooth the surface topography is required to be. The surface finish should be such that the scratches as well as their depth should be less than $10 \%$ of the diameter of the impression [2]. The specimen for nanoindentation were prepared by the resin mounting and subjected to metallographic polishing. Oxide polishing using $0.15 \mu \mathrm{m}$ Aluminium oxide suspension was the last polishing step. Two types of specimen were used: (1) plain wire specimen (2) Gold wire wirebonded to Silicon. Our aim during metallographic specimen preparation was to get a highly polished longitudinal cross section of the wire /wirebond.

Specially wirebonded samples were made in which a series of 5-10 gold wirebonds parallel to each other were bonded on a piece of silicon with $12 \mu \mathrm{m}$ thick film of gold sputter deposited on it. The free air ball diameter of the wirebonds was 60um (2.4 times wire diameter). Bondability of gold wirebonds is better on gold and hence gold thin films were deposited on silicon as a bonding surface. Since gold does not adhere well to silicon, a thin layer $(4 \mu \mathrm{m})$ of chromium was first deposited on silicon and then gold was deposited on top of it The bonding surface was not preheated. The wirebonded sample is mounted in curing resin such that the crosssection of the wirebond parallel to the wire axis is oriented parallel to the grinding surface. Since all wirebonds are parallel to one another, once a wirebond cross section is tested, it can be ground away to produce a new cross section from the adjacent next wirebond.

\section{WIRE COMPOSITION ANALYSIS: TOF-SIMS}

The gold bonding wire used throughout this research has a purity $4 \mathrm{~N}(99.99 \%)$. The total amount of impurities plus doping elements does not exceed 100 ppm. The doping elements are generally from alkali, alkaline earth and rare earth groups as well as those of group III and IV of the periodic table [3]. Actual composition of the wires however is not known since such knowledge is proprietary of the bonding wire producers which is why SIMS analysis was conducted on our gold sample.

As observed in the spectrum the elements detected with strong peaks are Beryllium, Sodium, Magnesium, Aluminium, Silicon, Potassium, Calcium, Scandium, Titanium and Iron were detected with very small peak intensities. Also Barium, Lanthanum and Cerium were detected but their peak intensities were extremely low enough to be neglected. And of course, Gold being most abundant, a sharp peak was observed at mass number 197. The doping elements influence the mechanical properties of the wire and recrystallization temperature and thus control the grain size and length of the heat affected zone.

The problem however is that with the help of TOF-SIMS only the presence of the elements could be detected but their quantities could not be ascertained due to reasons described below. Quantitative characterization using SIMS can be done only if reference materials with

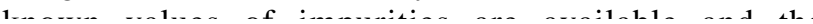

comparison can then be made between the peak intensities of impurity in the reference material and that in the unknown sample to estimate the quantity of a particular element in the unknown sample. However in absence of availability of such reference gold sample quantitative measurements could not be undertaken.

Next step after the identification of the dopants was to observe the distribution of the dopants in the grains and see segregation at the grain boundaries. This technique was first described and has been used with partial success by Donthu et al[4]. For this, two types of SIMS images are required, a secondary electron image and secondary ion image. The secondary ion image gives the position of dopant on the sample while the secondary electron image shows the grain structure of the specimen at that position. The secondary electron image is able to show crystallographic contrast since secondary electron output is dependent on the crystallographic orientation and each grain has different orientation because of which each grain looks different in secondary electron image. When we superimpose the secondary ion image depicting positions of the dopants and electron image showing grain structure, we can observe a distribution of dopants in the grains and observe if there is segregation of doping elements at the grain boundaries. Correlations can then be made between the mechanical properties in the heat affected zone and the dopant segregation to demystify and get a clear explanation of mechanism of doping induced improvement in mechanical properties and change the current "Add the dopant-Observe the effects" approach used by bonding wire manufacturers. But both secondary electron and ion image are needed to observe this. Secondary electron image requires specimen surface to be highly planar (much more than that required for nanoindentation) otherwise the surface roughness deflects the secondary electrons and no image is obtained. Our specimen being mechanically polished the surface roughness prevented the acquisition of suitable electron image and a proper image was not obtained to carry out this study using SIMS further. Besides this, the small dimension of the wirebond created additional problems in viewing the grain structure.

\section{WIRE RECRYSTALLIZATION CHARACTERISTICS - DIFFERENTIAL SCANNING CALORIMETRY (DSC)}

During the ball-bonding, when the tip of the wire is melted to form a ball, the heat is dissipated out of the ball, along the length of the wire. Gold being a good thermal conductor the heat loss from the ball is primarily through thermal conduction along the wire. The part of the wire immediately next to the ball experiences the temperature shooting up close to the melting point of gold $\left(1064^{0} \mathrm{C}\right)$ and gradually gets cooled back to the ambient temperature. Due to this, recrystallization and grain growth takes place in the heat-affected zone (HAZ) creating a disadvantageous coarse grain structure in HAZ which has lower strenoth as comnared to the rest of the 

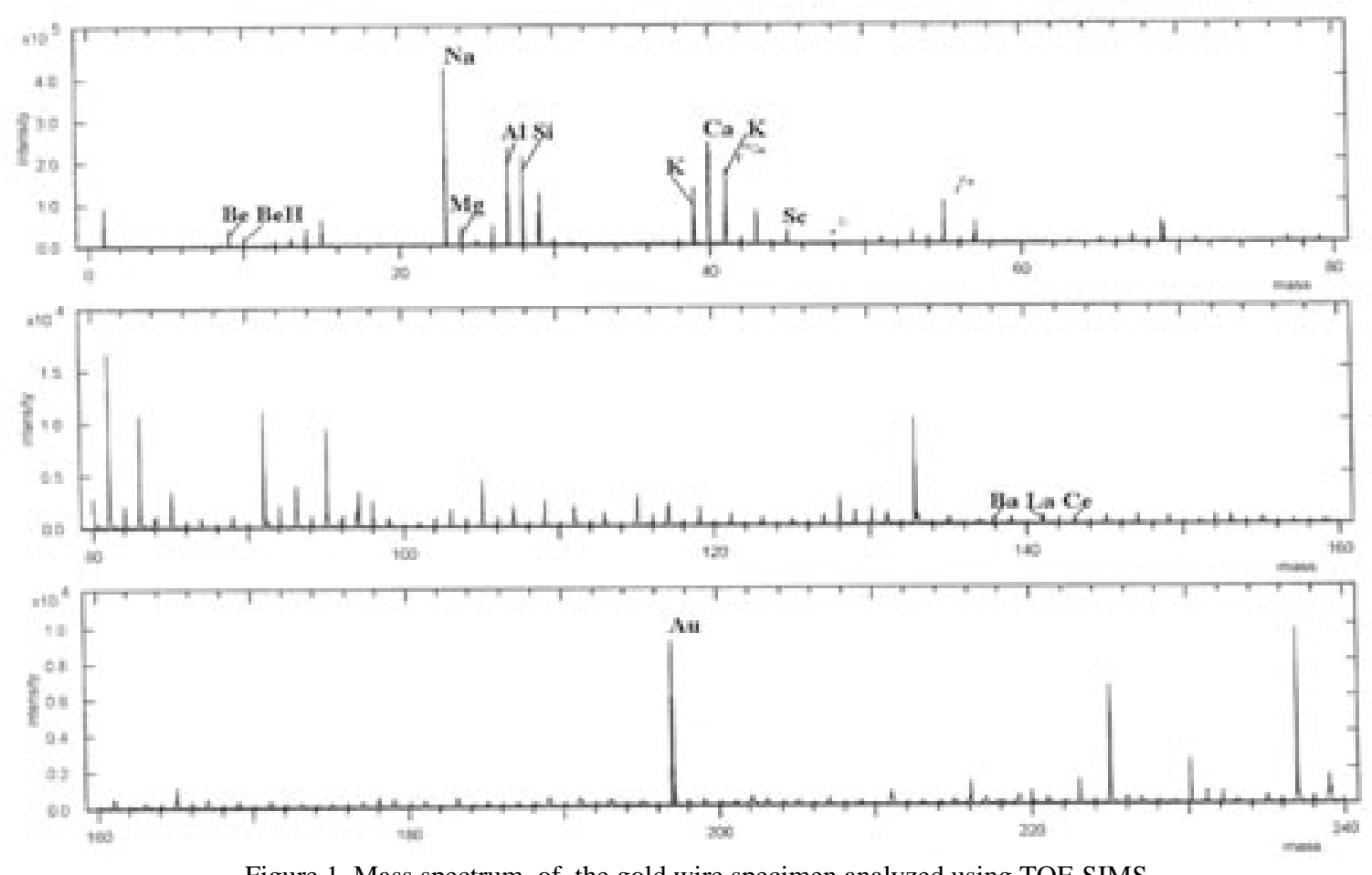

Figure 1 Mass spectrum of the gold wire specimen analyzed using TOF-SIMS

wirebond (Hall-Petch relationship). For a given ball size ratio, the lower the recrystallization temperature, the longer is the HAZ and hence higher the loop height. By measuring recrystallization temperatures using DSC and HAZ length using nanoindentation for different gold bonding wires (while keeping the ball size ratio constant), one can obtain an atlas of data showing a wire of what recrystallization temperature will give how long HAZ (and therefore the loop height). Here we have demonstrated this for one gold bonding wire that was used throughout this work.

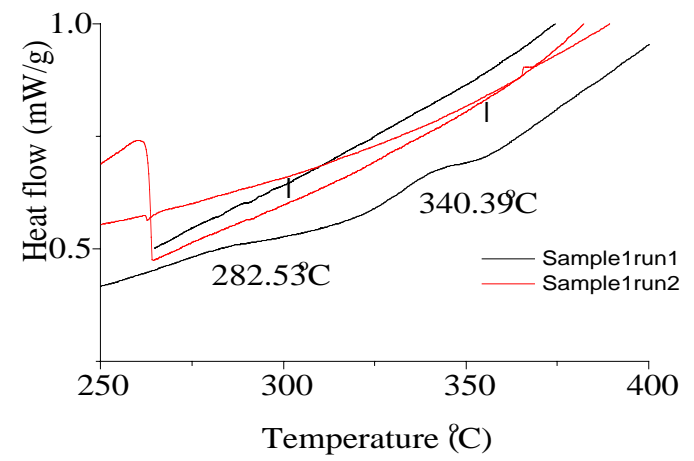

Figure 2 Graph depicting the heat flow during D.S.C. against temperature for the first gold wire specimen

Differential scanning experiments were carried out for two specimens of the same wire having weight of $17.7 \mathrm{mg}$ and $20.98 \mathrm{mg}$ respectively. The temperature wasraised at a constant preset rate of $5^{\circ} \mathrm{C} / \mathrm{min}$ until a maximum temperature of $550^{\circ} \mathrm{C}$ was reached. During this ramp-up, the difference in power required to keep the temperatures of the two holders equal was measured. A graph is plotted of this parameter against the

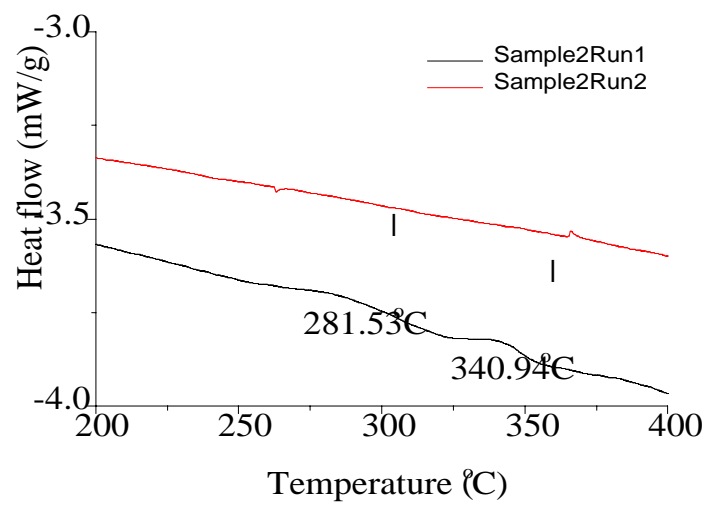

Figure 3 Graph depicting the heat flow during D.S.C. against temperature for the second gold wire specimen

temperature which is as shown below. Two peaks were observed in both cases as shown in figures 2 and 3. The first peak is presumed to be due to recover while the second peak corresponds to the recrystallization phenomena. The peaks for both the wire specimen match quite well and recrystallization is observed to occur at $340.39^{\circ} \mathrm{C}$ and $340.94^{\circ} \mathrm{C}$ respectively. Thus the average recrystallization temperature of gold wire used for ball bonding was found to be $340.66^{\circ} \mathrm{C}$.

\section{NANOINDENTATION TESTS AND ANALYSIS}

Nanoindentation tests were carried out using Ultra Micro Indentation System (UMIS-2000 H) developed by CSIRO Australia. The indentation tip of n ...1 . 1 
in the indentation equipment a time period of 30 minutes was allowed for temperature to stabilize in the enclosure. The outcome of the nanoindentation test is in the form of load-displacement curves from which the mechanical properties can be determined by relations below [5].

The "composite" or "reduced" modulus E* of contact is given by

$$
\mathrm{E}^{*}=\frac{1}{\mathrm{c} * \sqrt{\mathrm{A}}}\left(\frac{\mathrm{dP}}{\mathrm{dh}}\right)
$$

where $\mathrm{c}^{*}=1.167$ for Berkovich indenter, $\mathrm{A}$ is the contact area and $\mathrm{dP} / \mathrm{dh}$ is the slope of the unloading curve at maximum load.

and hardness $\mathrm{H}$ is given by

$$
\mathrm{H}=\frac{\mathrm{P}}{\mathrm{A}}
$$

The Elastic modulus of the specimen material $\mathrm{E}_{\mathrm{m}}$ can be obtained from the composite modulus using the relation

$$
\frac{1}{\mathrm{E}^{*}}=\frac{\left(1-v_{i}^{2}\right)}{\mathrm{E}_{\mathrm{i}}}+\frac{\left(1-v_{\mathrm{m}}^{2}\right)}{\mathrm{E}_{\mathrm{m}}}
$$

where $v$ is the poisson's ratio and E is the young's modulus and the subscripts $\mathrm{i}$ and $\mathrm{m}$ refer to indenter and specimen materials respectively, $\mathrm{E}_{\mathrm{i}}=1000 \mathrm{GPa}$ and $v_{\mathrm{i}}=0.07$ for diamond indenter, $v_{\mathrm{m}}=0.42$ for gold [6].

The contact area A for the equations (1) \& (2) according to Oliver and Pharr model is

$$
\mathrm{A}=24.5 \mathrm{~h}_{\mathrm{c}}{ }^{2}
$$

where $h_{c}$ is the contact depth given by

$$
\mathrm{h}_{\mathrm{c}}=\mathrm{h}_{\max }-\varepsilon\left(\mathrm{h}_{\max }-\mathrm{h}_{\mathrm{i}}\right)
$$

where $\varepsilon=0.72$ for Berkovich indenter, $h_{\max }$ is the maximum penetration depth and $h_{i}$ is the intercept of the slope of unloading curve on abscissa.

\section{RESULTS AND DISCUSSION}

An ideal load-depth curve obtained from a single load-unload type indentation should be parabolic in nature however nanoindentation on our gold wirebond specimen showed clear deviation from parabolic nature in the initial loading stages and the curve became parabolic only after penetration of a certain finite depth. It is presumed to be due to the presence of a small residual work hardened layer on the surface introduced during mechanical polishing. It is therefore expected that for a mechanically polished surface, a single load-unload indentation would not yield accurate value of hardness. Therefore indentations were carried out in the multiple load-partial unload mode. The indentation carried out in this mode gives values of hardness and elastic modulus at various depths at a single point of indentation. These values are then plotted against the depth of penetration of indenter to observe the trend. Indentations in this mode were carried out at various maximum loads: $3 \mathrm{mN}, 5 \mathrm{mN}, 7 \mathrm{mN}, 9 \mathrm{mN}$, $11 \mathrm{mN}, 15 \mathrm{mN}$ and $20 \mathrm{mN}$ to observe the effect of use of higher loads on values of hardness. At least 5 indentation

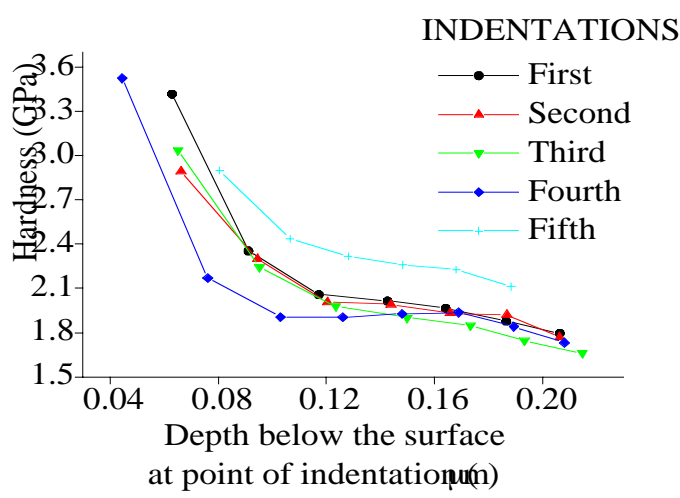

Figure 4 Hardness variation with depth for $3 \mathrm{mN}$ maximum load

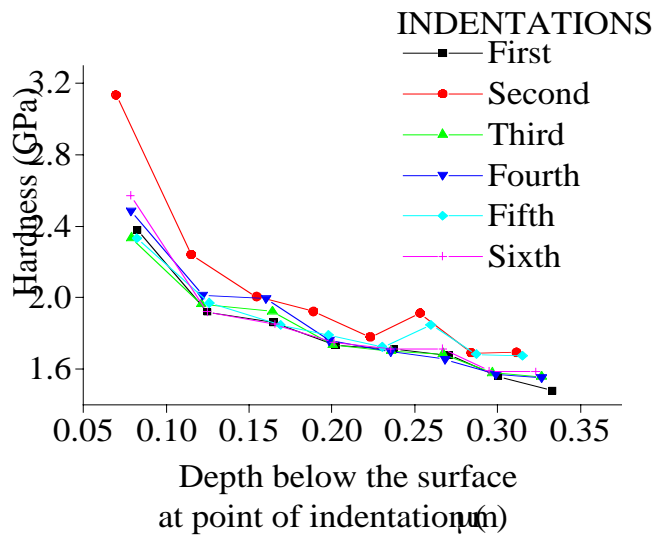

Figure 5 Hardness variation with depth for a $5 \mathrm{mN}$ maximum load

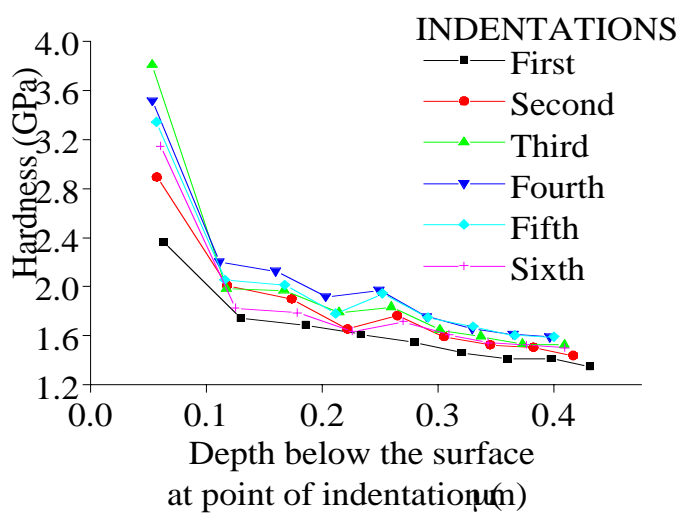

Figure 6 Hardness variation with depth for $7 \mathrm{mN}$ maximum load

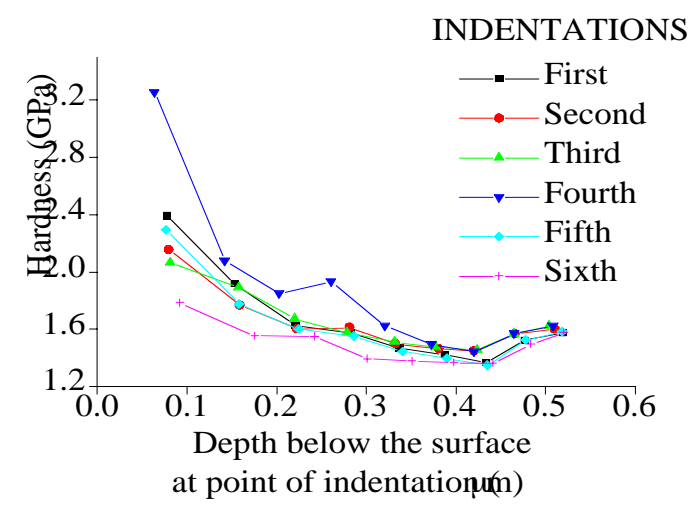

INDENTATIONS 


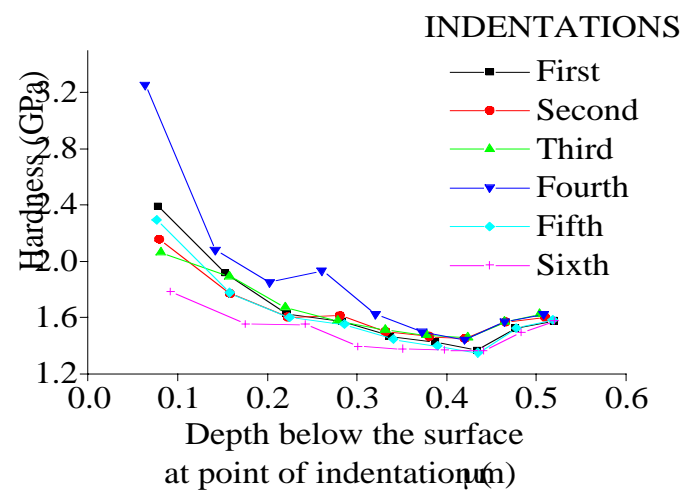

Figure 8 Hardness variation with depth for $11 \mathrm{mN}$ maximum load

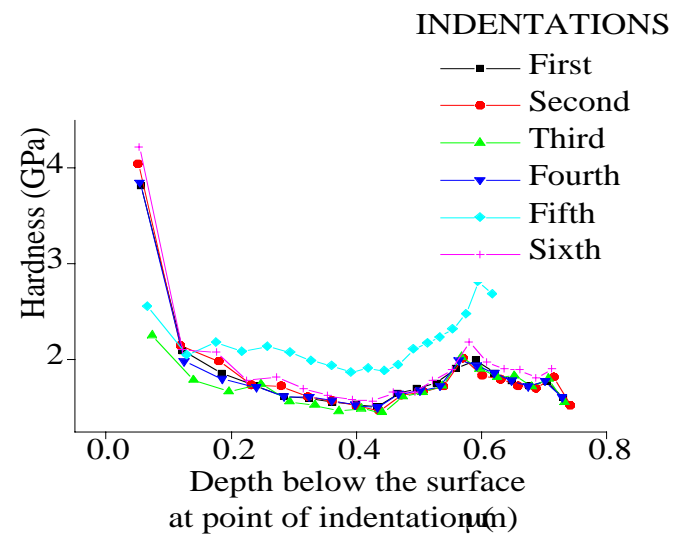

Figure 9 Hardness variation with depth for $15 \mathrm{mN}$ maximum load

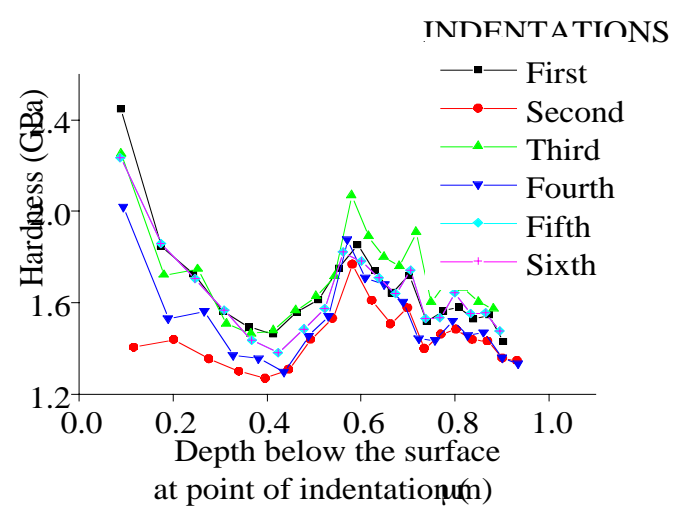

Figure 10 Hardness variation with depth for $20 \mathrm{mN}$ maximum load

the nature of the data obtained. These are presented in figures 4 to 10 .

All the graphs, irrespective of the maximum load, show the hardness values to be decreasing from maximum at the surface to a uniform value at a certain depth into the specimen. It is also observed that with lower loads, $3 \mathrm{mN}$, $5 \mathrm{mN}$ and $7 \mathrm{mN}$ the maximum load is reached before the hardness value would stabilize and hence the maximum load is insufficient to determine the actual hardness. Hardness values become consistent at higher depths of penetration which require higher maximum loads. For loads $9 \mathrm{mN}$ and $11 \mathrm{mN}$, the hardness values decrease until they become uniform and remain constant upto a certain depth and then start rising again. Once the hardness values rise, they peak and fall repeatedly for higher loads $15 \mathrm{mN}$ and $20 \mathrm{mN}$. The gold wire specimen is only $25 \mu \mathrm{m}$ wide so after the penetration beyond certain depth, the mounting resin surrounding the gold wire influences the indenter tip which is why the hardness values start rising from the consistent readings. These readings are ignored and only the uniform readings are averaged out to get the final value of hardness. The hardness value of gold wire was determined to be $1.41 \mathrm{GPa}$ at $9 \mathrm{mN}, 1.52 \mathrm{GPa}$ at $11 \mathrm{mN}$, $1.56 \mathrm{GPa}$ at $15 \mathrm{mN}$ and $1.35 \mathrm{GPa}$ at $20 \mathrm{mN}$. It was also observed that a load of $9-11 \mathrm{mN}$ is sufficient for indentation of polished gold wire of the said dimensions. Loads lower than this fail to produce uniform readings and higher loads only produce readings with edge influence after a certain depth.

The reason for higher hardness values registered at initial lower loads can be due to two reasons: one, because of the so-called "indentation size effect", two, because of the presence of work hardening in the subsurface region. Indentation size effect suggests that for the same material, the hardness value depends on the size of the indentation and hence on the load used, the lower the load used for indentation, the higher the hardness value. At lower loads, smaller volumes of material are stressed which might be effectively free from crystal defects and so the most of the stress is consumed by the formation of dislocation loops [7]. The hardness values have been experimentally measured to approach theoretical strength of material[8] at very low loads. Added to this, is the work hardening of the surface due to the mechanical polishing procedure employed for surface preparation. These two effects superimpose on each other to produce the higher hardness profile at initial loading stages in the curves presented in figures 4 to 10 .

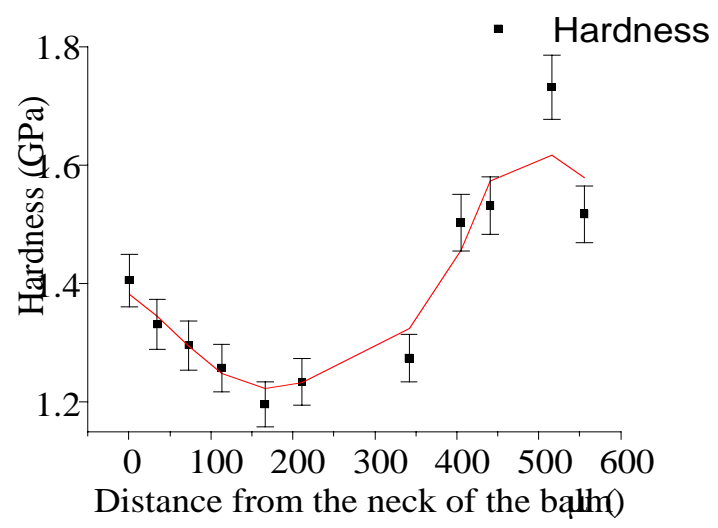

Figure 11 The hardness variation with distance from the neck of the ball in gold wirebond

After testing the plain gold wire with different loads, a mechanically polished gold wirebond cross section was subjected to a series of nanoindendations along the length of the wirebond starting from the neck greion next to the ball, with $10 \mathrm{mN}$ maximum load. The indentations were spaced 20-30 microns apart to keep them from influencing one another. The hardness values obtained from these nanoindentations are plotted against the distance of indentation point from the ball neck as 
It is observed that the hardness values tend to decrease with the distance from the ball neck until it reaches a minimum value at around $166 \mu \mathrm{m}$, beyond which it is observed to rise rapidly until it reaches the hardness value of the heat unaffected wire. The general increase in hardness values away from the ball, as observed, can be explained to be due to grain size effect. As seen in the microstructure in figure 12 the grain size gradually decreases away from the ball towards the heat unaffected wire. The plastic properties like hardness and yield strength improve as the grain size decreases (Hall-Petch behaviour) and their dependence on grain size is given by

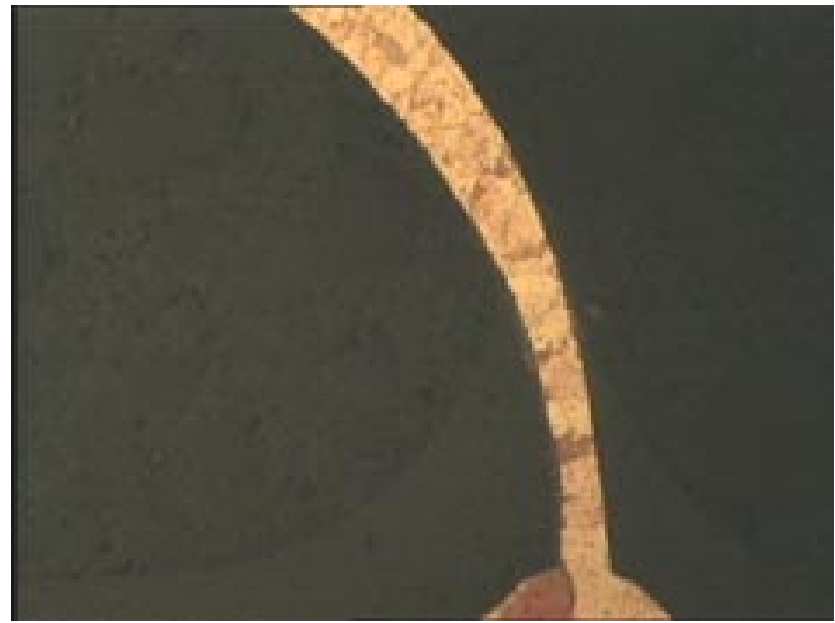

Figure 12 Microstructure of Heat affected zone showing grain size distribution $(500 \mathrm{X})$

the Hall-Petch equation: $\sigma_{\mathrm{y}}=\sigma_{0}+\mathrm{kd}^{-1 / 2}$. So the increase in hardness with decrease in the grain size is justified. But in the region next to the ball neck, the hardness has been observed to decrease upto a distance of around $166 \mu \mathrm{m}$ showing hardness decreasing with decreasing grain size, which is anti-Hall-Petch behaviour. The explanation of this observation lies in the thermal history of the neck region [9].

During the formation of the ball by melting of the tip of wire the temperature in the neck region shoots to very high temperatures close to the melting point of Gold $\left(1064^{\circ} \mathrm{C}\right)$ and then drops rapidly to the room temperature as the heat is conducted away from this region to the rest of the gold wire. According to Thermodynamics, at every temperature there is a corresponding equilibrium number of vacancies present in a material. The higher the temperature, the higher is the number of vacancies present. So when the neck region heats upto a temperature close to the melting point, there is an equilibrium number of vacancies corresponding to that temperature present. As the material cools down, the excess vacancies diffuse through the material and escape to the free surfaces or grain boundaries and/or get annihilated by other mechanisms. However in the neck region the material is rapidly quenched from a very high temperature and sufficient time is not available for the excess vacancies to diffuse through. So there are trapped quenched-in excess vacancies present in the neck region. Meshii and Kaufman [10] who have studied quench hardening in pure gold random stable clusters of vacancies which act as barriers to dislocation motion thus contributing to increase in hardness. When these two effects, quench hardening and the grain size effect superimpose, they create the v or ushaped hardness profile as observed.

The elastic modulus values were found to be varying randomly between 40-60 GPa. The bulk elastic modulus of gold in absence of any texture is $77.2 \mathrm{GPa}$ [11]. The random variation is because of the orientation dependence of the Elastic modulus of Gold crystal. Gold is highly anisotropic. No trend was observed in Elastic modulus values obtained from the various points on the wirebond. The elastic modulus of a material being a "structureinsensitive" property [12] does not depend on the microstructure of a material unlike the plastic properties like Hardness and Yield strength.

The yield strength values can be estimated from the measured hardness values using the correlation between hardness and yield strength i.e. $H=3 \mathrm{Y}$ [13]. So accordingly the expected variation of yield stress is as shown in figure 13 .

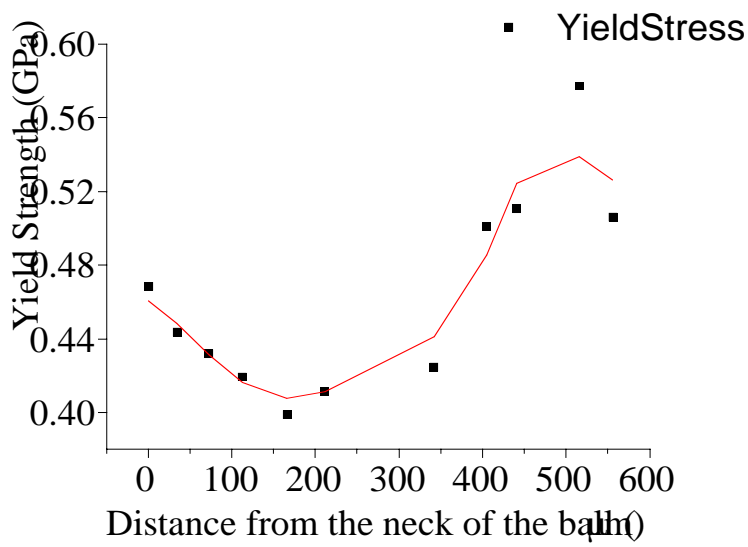

Figure 13 The suggested yield strength variation with distance from the neck of the ball

As in the case of hardness the yield strength is also minimum at the same point where the hardness is minimum. The material at this point is first to yield during the wirebonding process and would witness maximum deformation. But the use of such hardness and yield strength correlation may not predict very accurate values of yield strength since such correlations are more accurate when material is isotropic which is not so in the present case. This can be the biggest source of error in this prediction. However in absence of any methods for direct measurement of yield stress using nanoindentation, use of such method for estimation of yield strength is the only available alternative.

\section{CONCLUSION}

We have mechanically characterized the gold wire of $25 \mu \mathrm{m}$ diameter and wirebond made from the same with a ball size ratio of 2.4 using nanoindentation details of which are also in [14]. The doping elements in the gold 
however dopant distribution in the grains in HAZ could not be observed for correlation between dopant segregation and mechanical properties measured using nanoindentation. The recrystallization temperature of the wire used was determined using D.S.C. to be $340.66^{\circ} \mathrm{C}$. The specimen surface was prepared by mechanical polishing till $0.15 \mu \mathrm{m}$ oxide polishing achieved the required surface planarity but on the flip side introduced a finite depth of subsurface deformation which along with indentation size effect became a source of higher hardness values at lower penetration depths. Loads in the range 9$11 \mathrm{mN}$ were found to be suitable for characterization of mechanically polished gold wirebond of the said dimension. A v-shaped hardness profile was observed along the HAZ of the gold wirebond for $10 \mathrm{mN}$ maximum load controlled indentation for which the microstructure of the wirebond is responsible. For a gold wire of recrystallization temperature $340.66^{\circ} \mathrm{C}$ the wirebond with a ball size ratio 2.4 was found to have heat-affected zone (HAZ) length of $166 \mu \mathrm{m}$. No specific trend was observed in the elastic modulus values along the HAZ. An approximate trend of yield stress variation along the HAZ of the gold wirebond is obtained using a hardness-yield strength correlation for isotropic materials.

\section{ACKNOWLEDGEMENTS}

One of the authors, M.Shah would like to express his gratitude to Singapore-MIT Alliance (SMA) for the financial assistance provided to him during the course of research. We would also like to thank the Institute of Materials Research and Engineering (IMRE), a research institute affiliated to National University of Singapore (NUS) for the equipment support.

\section{REFERENCES}

[1] A.A.O.Tay, B.C.Seah, S.H.Ong, "Finite element simulation of wire looping during wirebonding", Advances in Electronic Packaging 1997. Proceedings of the Pacific Rim/ASME International Intersociety Electronic and Photonic Packaging Conference. INTERpack '97, vol 1, pp. 399-406, 1997

[2] M.C.Shaw, Science of hardness testing and its research applications, ed. J.H.Westbrook, H.Conrad, pp. 1-15

[3] Ch Simons, L Shrapler and G.Herklotz, "Doped and low-alloyed gold bonding wires", Gold Bulletin, vol. 33, no.3, 2000, vol. 33, no. 3, pp. 89-96, 2000

[4] S.K.Donthu, Self annealing in electroplated copper films, M.Eng. thesis, Singapore-MIT Alliance, National University of Singapore, 2001

[5] S.P.Baker, "The Analysis of depth-sensing indentation data", Materials Research Society symposium proceedings, vol 308, pp. 209-216, 1993

[6] Properties and Selection: Non ferrous alloys and special purpose materials, Metals handbook, ASM international, $10^{\text {th }}$ Ed., 1990

[7] S.Suresh, T.G. Nieh and B.W.Choi, "Nanoindentation of Copper thin films on Silicon substrate", Scripta
[8] N.Gane, Proc. Roy. Soc. (London), 317A, 367(1910)

[9] Ohno Y. et al, "Factors governing the loop profile", 1992 Proceedings. 42 ${ }^{\text {nd }}$ Electronic Components and Technology Conference, pp. 899-902, 1992

[10] M.Meshii, J.W.Kaufman, "Quenching studies on mechanical properties of pure gold", Acta Metallurgica, vol 7, march, pp. 180-186, 1959

[11] M.Pecht, Electronic packaging materials and their properties, pub. CRC press, 1998

[12] G. Dieter, Mechanical Metallurgy, pub. McGraw Hill, 1988

[13] D.Tabor, The hardness of metals, Oxford press, 1951

[14] M.Shah, Measurement of mechanical properties of gold wirebonds used in ICs using nanoindentation, M.Eng. thesis, Singapore-MIT Alliance, National University of Singapore, 2001

$*$ * $111 \cdot$ n ner ner $1 \mathrm{nnn}$ 